\title{
Operating Conditioning in Bennett Ualabies (Macropus Rufogriseus) Ex Situ for Drugs Administration by Oral Route
}

By Martínez-Espina, Verónica Isabel, Castañeda Díaz-Samayoa, Andrea \& Villatoro-Chacón, Daniela Mariel

Universidad de San Carlos de Guatemala

Abstract- The objective of this study was the oral administration of trimetoprim sulfamethoxazole as a prophylactic therapy in Bennett's Wallabies (Macropus Rufogriseus) through the use of operant conditioning. In the study participated 15 animals of different gender and age, all of them living at "La Aurora" National Zoo in Guatemala City, Guatemala. The investigation was divided in 2 stages, first the desensitization and conditioning of the animals and second the oral administration of the drug. Results indicated that $76 \%$ of the population had a positive response to the desensitization and conditioning, however only $20 \%$ consumed the $100 \%$ of the dose. In conclusion the operant conditioning is an excellent tool for desensitizing and creating a routine with zoo animals, but further investigation is required to determine the efficiency of the administration of an oral treatment on this species.

Keywords: conditioning, sensitization, stimulus.

GJMR-G Classification: NLMC Code: WA 360

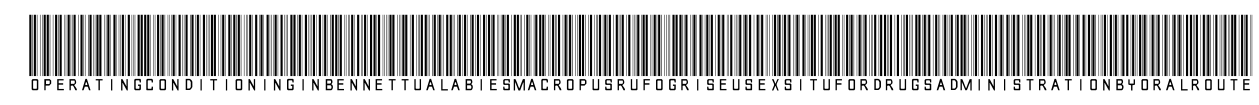

Strictly as per the compliance and regulations of:

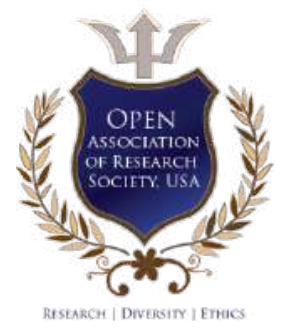

(c) 2021. Martínez-Espina, Verónica Isabel, Castañeda Díaz-Samayoa, Andrea \& Villatoro-Chacón, Daniela Mariel. This is a research/review paper, distributed under the terms of the Creative Commons Attribution-Noncommercial 3.0 Unported License http://creativecommons.org/licenses/by-nc/3.0/), permitting all non-commercial use, distribution, and reproduction in any medium, provided the original work is properly cited. 


\title{
Operating Conditioning in Bennett Ualabies (Macropus Rufogriseus) Ex Situ for Drugs Administration by Oral Route
}

\author{
Condicionamiento Operante En Ualabies De Bennett (Macropus Rufogriseus) Ex \\ Situ Para La Administraciónde Fármacos Por Vía Oral
}

Martínez-Espina, Verónica Isabel ${ }^{\alpha}$, Castañeda Díaz-Samayoa, Andrea ${ }^{\sigma}$ \& Villatoro-Chacón, Daniela Mariel ${ }^{p}$

\begin{abstract}
Resumen- El objetivo de este estudio fue el uso de condicionamiento operante para la administración oral de trimetoprim sulfametoxazol como terapia profiláctica para toxoplasmosis en los Ualabies de Bennett (Macropus Rufogriseus). En el estudio participaron 15 ejemplares de diferente sexo y edad que conforman el grupo de animales del Parque Zoológico Nacional La Aurora en la ciudad de Guatemala, Guatemala. La investigación se realizó en 2 etapas, siendo la primera, la desensibilización y condicionamiento de los ejemplares y la segunda la administración oral del medicamento. Los resultados mostraron que el $76 \%$ de la población obtuvo un resultado positivo a la desensibilización y condicionamiento, pero sólo el $20 \%$ de la población consumió el $100 \%$ de la dosis calculada en el estudio. Por tanto, se concluye que el condicionamiento operante es una excelente herramienta para la desensibilización y habituación de los animales a una rutina establecida, pero se requieren estudios más extensos para determinar su eficacia en cuanto a la administración oral de un tratamiento profiláctico en esta especie.
\end{abstract}

Palabras clave: condicionamiento, sensibilización estímulo.

Abstract- The objective of this study was the oral administration of trimetoprim sulfamethoxazole as a prophylactic therapy in Bennett's Wallabies (Macropus Rufogriseus) through the use of operant conditioning. In the study participated 15 animals of different gender and age, all of them living at "La Aurora" National Zoo in Guatemala City, Guatemala. The investigation was divided in 2 stages, first the desensitization and conditioning of the animals and second the oral administration of the drug. Results indicated that $76 \%$ of the population had a positive response to the desensitization and conditioning, however only $20 \%$ consumed the $100 \%$ of the dose. In conclusion the operant conditioning is an excellent tool for desensitizing and creating a routine with zoo animals, but further investigation is required to determine the efficiency of the administration of an oral treatment on this species.

Keywords: conditioning, sensitization, stimulus.

Author a o: Parque Nacional Zoológico La Aurora, Guatemala, ciudad. Author p: Universidad de San Carlos de Guatemala, Facultad de Medicina Veterinaria y Zootecnia, Departamento de Ayudas Diagnósticas, Hospital Veterinario.

e-mail: danavilla47@profesor.usac.edu.gt

\section{INTRODUCCIÓN}

I Parque Zoológico Nacional La Aurora, se encuentra en la ciudad de Guatemala y cuenta con una población de alrededor de 3,000 ejemplares, siendo el único zoológico del país que cuenta con Ualabies de Bennett (Macropus rufrogriseus). Debido a su característica de presa en su hábitat natural, el manejo médico de estos animales es un proceso estresante tanto para el ejemplar como para el personal de los zoológicos. Esto ha obligado al personal encargado de fauna silvestre a implementar métodos que reduzcan el estrés en los animales como lo es el condicionamiento operante (Yin, 2006).

El condicionamiento operante, es una técnica que se ha utilizado en diversas especies como linces (Crowell, 2008), venados (Crowell, 2008), tortugas aldabras (Weiss, 2003), primates del nuevo mundo (Savastano, 2003), iguana cabeza roja(Hellmuth, 2012), cocodrilo del Nilo(Hellmuth, 2012), ñacanina (Hellmuth, 2012), rinocerontes blancos y negros (Holden, 2006), babuinos (Martina, 2020) y pitón de Birmania (Emer, 2015). En Latinoamérica se han realizado estudios en tigres (Gomez, 2015)(Damian, 2016), chimpancés (Hincapié, 2019), león africano (Damian, 2016), león blanco (Damian, 2016), rinoceronte blanco (Molina, 2018)(Hoyos, 2017) jirafas (Uribe, 2019) y jaguar (Jácome, 2012)(Guzmán, 2018).En Centroamérica se ha reportado en zarigüeyas americanas (James, 1937)(Ravizza, 1969) y (Cheney, 1980), sin embargo, (Panini, 1986)menciona que estos estudios realizados en zarigüeyascarecen de bases sólidas para indicar la efectividad del condicionamiento operante en estos animales, ya que las respuestas presentadas por los ejemplares de los estudios pueden ser un acto de sensibilización a los estímulos aberrantes realizados en estas investigaciones.

El presente estudio genera información respecto al condicionamiento operante en Macropus rufrogriseus como una herramienta que permite el 
manejo médico de la especie para su conservación en cautiverio.

\section{Métodos}

\section{a) Área de estudio}

El Zoológico La Aurora está ubicada en el Boulevard Juan Pablo II, 5ta calle interior Finca "La Aurora", Ciudad de Guatemala, zona 13. Se encuentra frente al aeropuerto nacional La Aurora, entre el boulevard Liberación y el mercado de artesanías. Sus coordenadas geográficas son 14037'16" latitud norte y $90^{\circ} 31^{\prime} 37^{\prime \prime}$ longitud oeste. La ciudad de Guatemala se encuentra a 1,592 metros sobre el nivel del mar. La temperatura oscila entre 12 y 18 grados centígrados. La humedad relativa es de 64\% a 84\%.(Ordóñez, 2012).

\section{b) Población del estudio}

El grupo de Ualabies cuenta con 15 ejemplares, de los cuales se cuenta con 4 machos enteros (de 14 a 17 kg.), 9 hembras enteras (de 10 a 12 kg.) y 2 crías (de $4 \mathrm{~kg})$.

\section{c) Hábitat}

Los recursos utilizados fueron el recinto de los ualabies de Bennett y canguros rojosque contiene el recinto de exhibición, recinto de cacatúa, patio trasero, dormitorio para canguros y dormitorio para ualabies compuesto de dos áreas (figura 1). Los animales son alimentados con concentrado comercial Leaf eater ${ }^{\circledR}$, lechuga, zanahoria rallada, manzana, pellets de alfalfa y agua ad libitum.

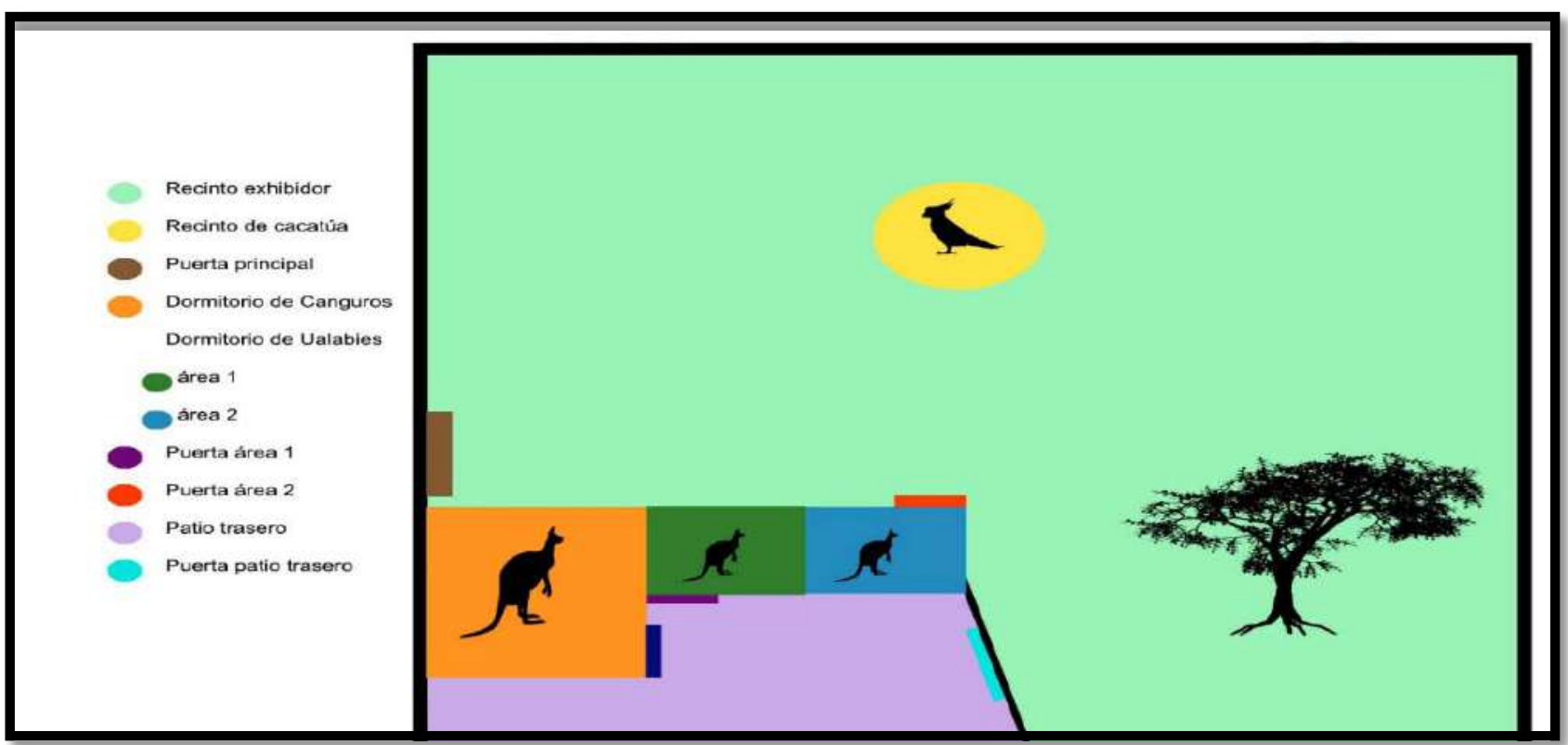

Figura 1: Mapa del recito de los ualabies de Bennett

\section{d) Periodo de desensibilización}

El período de desensibilización se realizó en 4 mesesabarcando las etapas de entrenamiento y condicionamiento, y consistió en 2 entrenamientos diarios de 1 hora cada uno aproximadamente, 5 días a la semana (lunes a viernes).

Como parte de la desensibilización se inició con el ofrecimiento del concentrado para su consumo desde la mano del cuidador o entrenador. Se ofreció una pieza de concentrado en cada intento, ya que estos animales consumen su alimento a una velocidad moderada, por lo que el ofrecimiento de varias piezas no presentaba ningún beneficio en la mayoría de ejemplares, siendo la excepción, los machos alfa, quienes consumían el concentrado con mayor rapidez. Luego de que esta acción fuera aceptada se continuó con el contacto físico con el animal, este se inició de forma gradual empezando por tocarle la cabeza, luego la espalda y por último la cola. Este proceso fue realizado de forma gradual y con paciencia ya que no todos los ejemplares presentan el mismo grado de confianza debido a diversos factores, incluyendo tratamientos previos, edad, sexo, conducta propia de la especie y personalidad individual de cada espécimen. El tipo de manejo que fue utilizado fue el contacto libre, es decir sin ningún tipo de barrera de restricción, ya que, explica Torres (2004 en Martínez, 2016) es el método comúnmente utilizado con especies que no representan un gran peligro para el entrenador.

\section{e) Entrenamiento y condicionamiento}

Para el entrenamiento y condicionamiento se requirió del recurso humano (un entrenador, 2 cuidadores habituales de los animales y 2 sustitutos los días de descanso de los cuidadores principales).

Se realizaron dos entrenamientos:

- El primer entrenamiento se realizó a las 7 horas con los animales dentro de su dormitorio. El entrenador y uno de los cuidadores procedían a colocarse en la salida del dormitorio hacia el patio trasero, cuya 
puerta se encontraba abierta. Haciendo uso del alimento comercial, se inició abriendo la puerta corrediza del dormitorio al patio trasero, permitiendo el paso de un animal a la vez. Al momento de salir, al animal se le ofrecía inmediatamente una pieza el concentrado con la finalidad de realizar el refuerzo positivo de la acción de salir y la desensibilización de los individuos a la interacción con el entrenador.

- El segundo entrenamiento se realizó a las 9 horas con los animales en su recinto de exhibición. Con las puertas del dormitorio y el patio trasero cerradas, se procedía a colocar dos comederos dentro del área 1del dormitorio, luego se adicionó una mano del concentrado incluido en la dieta del grupo a cada comedero.

El cuidador se posiciona en la salida del dormitorio al patio trasero, cerrando la puerta corrediza del dormitorio nuevamente, mientras que el entrenador haciendo uso de el silbato y la caja de dieta, llama a los ualabies desde la puerta principal del área 2del dormitorio. Luego de realizar el llamado el entrenador debe retroceder de la puerta para permitir el ingreso de los animales durante 3 minutos. Al terminar el tiempo, el entrenador debe salir por la puerta principal del área 2 del dormitorio y cerrar la puerta. De esta forma los ejemplares quedan de nuevo encerrados en el dormitorio.

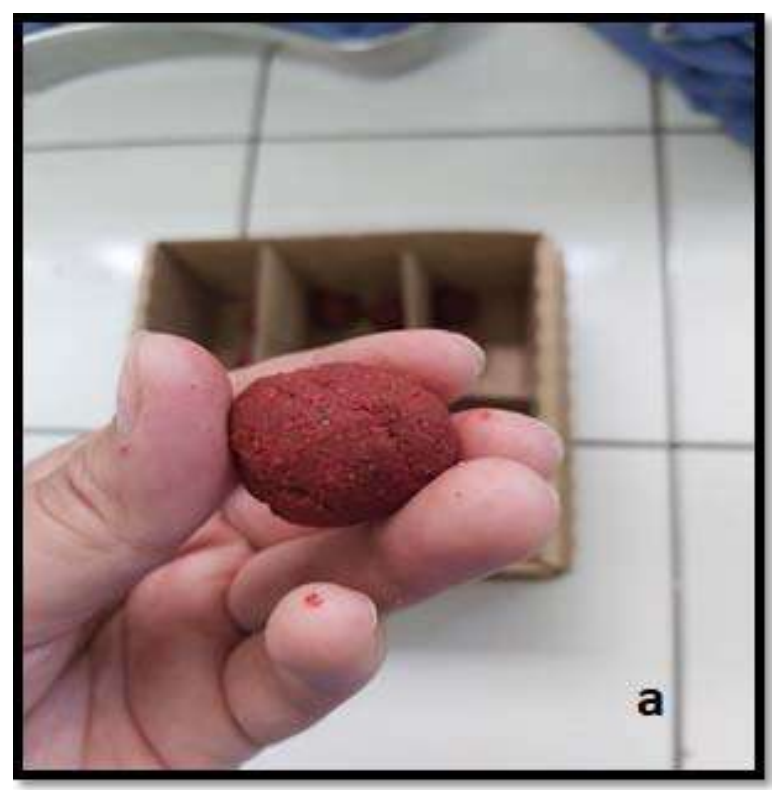

a. Leaf eater pulverizado mezclado con agua

b. Esferas de concentrado Leaf eater y trozos de pastilla de trimetoprima-sulfametoxazol

Figura 2: Esfera de concentrado Leaf eater pulverizado mezclado con agua

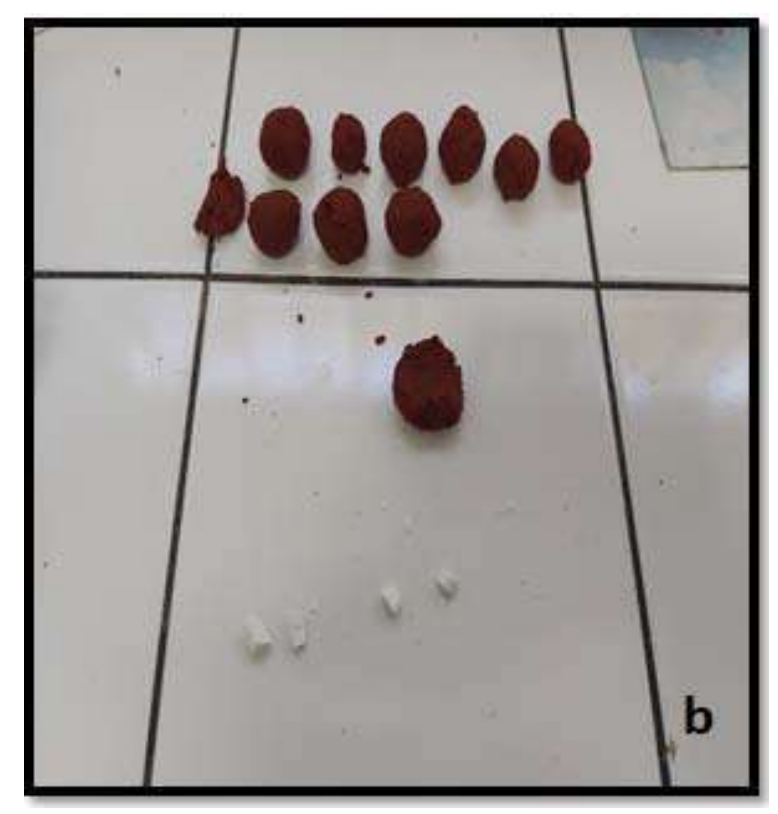

Cuando los ualabies se terminan la dieta de los comederos, se repite el entrenamiento de las 7 horas con ayuda del cuidador, permitiéndoles la salida del dormitorio hacia el patio trasero y luego al recinto de exhibición donde se administra la mitad de la dieta asignada para los ejemplares.

\section{f) Administración del tratamiento}

Como parte del cuidado profiláctico de estos animales se tiene establecido la administración de trimetoprim/sulfa para toxoplasmosis. Se utilizaron tabletas de $960 \mathrm{mg}$ de trimetoprima sulfametoxazol. Se utilizó una dosis de $20 \mathrm{mg} / \mathrm{kg}$ cada 24 horas por 10 días. Así pues, para los animales de $17 \mathrm{~kg}$ la dosis fue de $0.4 \mathrm{mg}, 14 \mathrm{~kg}$ a $0.3 \mathrm{mg}, 12 \mathrm{~kg}$ a $0.3 \mathrm{mg}, 10 \mathrm{~kg}$ a 0.2 mg y $4 \mathrm{~kg}$ a $0.1 \mathrm{mg}$

El medicamento fue colocado en pequeñas esferas del tamaño del concentrado y fueron pulverizadas y mezcladas con agua para colocarlas en los espacios seleccionados por el peso del animal (figura 2). La administración de las esferas con medicamento se realizó a las 7am, ya que, gracias al ayuno por la noche, eran más propensos a aceptar la medicación fácilmente (figura 3). El cuidador iniciaba sacando a los animales como de costumbre, por la puerta corrediza del área del dormitorio hacia el patio trasero y el entrenador ofrecía el alimento al animal 3 veces. 


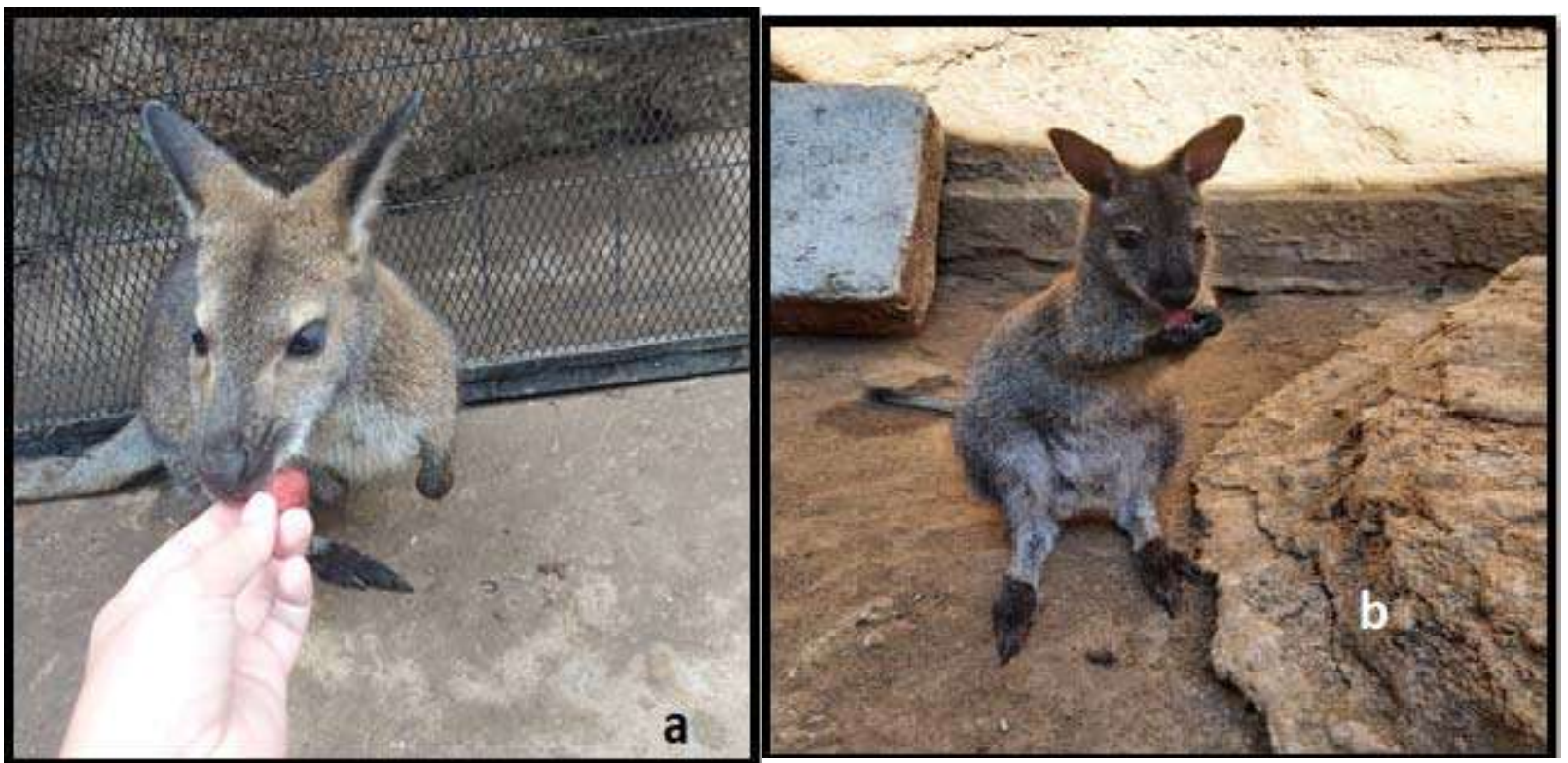

a. Ualabí hembra recibiendo el preparado de concentrado y medicamento

b. Ualabí cría recibiendo concentrado Leaf eater

Figura 3: Ualabí recibiendo concentrado Leaf eater

\section{g) Periodo de evaluación}

El periodo de evaluación de la investigación consistió en dos períodos: el período de desensibilización que tuvo una duración de 4 meses y el período de tratamiento que se realizó en 10 días consecutivos. En este último se administró el medicamento oral a los ejemplares. Durante el período de tratamiento se tomaron como válida la administración del medicamento en los primeros 3 ofrecimientos de la mezcla del concentrado medicado. Si el ejemplar no había consumido la dosis, esta se tomaba como un resultado negativo, aunque se le administrara el medicamento después. Se consideró un resultado positivo a la desensibilización a la población que acepto el contacto físico y a la presencia de los cuidadores y entrenador dentro del recinto; siendo negativo en los individuos que no lo aceptaran. Se consideró un positivo en la aceptación del medicamento a los individuos que aceptaran el 100\% de la dosis; siendo un resultado negativo los individuos que no consumieran la totalidad de la dosis.

\section{h) Análisis de datos}

Los resultados obtenidos fueron resumidos utilizando estadística descriptiva utilizando cuadros para su mejor análisis.

\section{ili. Resultados}

La desensibilización haciendo uso del condicionamiento operante el $76 \%$ de la población tuvo la aceptación de las acciones realizadas, considerándose un resultado positivo. En la tabla 1 se describen las distintas acciones y su aceptación por la población de estudio.

Tabla 1: Periodo de desensibilización de Macropus rufrogriseus

\begin{tabular}{ccc}
\hline Acción & $\%$ & $\mathrm{n}$ \\
\hline Aceptar el alimento & 100 & 15 \\
Aceptar alimento sin retirarse & 80 & 12 \\
Contacto físico en la cabeza & 80 & 12 \\
Contacto físico en la espalda & 66.7 & 10 \\
Contacto físico en la cola & 53.3 & 8 \\
\hline
\end{tabular}

Respecto a la aceptación del tratamiento, sólo el $20 \%$ de la población total mostró un resultado positivo al ingerir la totalidad de la dosis administrada. En la tabla 2, se describe el porcentaje de aceptación de cada dosis en cada individuo. 
Tabla 2: Aceptación de la dosis administrada en Macropus rufrogriseus

\begin{tabular}{ccc}
\hline Aceptación del tratamiento & $\%$ & $\mathrm{~N}$ \\
\hline Aceptación 100\% dosis & 20 & 3 \\
Aceptación 90\% dosis & 20 & 3 \\
Aceptación 80\% dosis & 13.3 & 2 \\
Aceptación 70\% dosis & 0 & 0 \\
Aceptación 60\% dosis & 6.68 & 1 \\
Aceptación 50\% dosis & 13.3 & 2 \\
Aceptación 40\% dosis & 6.68 & 1 \\
Aceptación 30\% dosis & 6.68 & 1 \\
Aceptación 20\% dosis & 6.68 & 1 \\
Aceptación 10\% dosis & 0 & 0 \\
Aceptación 0\% dosis & 6.68 & 1 \\
\hline
\end{tabular}

\section{Discusión}

En el proceso de desensibilización de los animalesex situ indican que el condicionamiento operante es una herramienta útil para crear un nivel de confianza entre el entrenador/cuidador y los ejemplares de una colección (Mattinson, 2012). Sin embargo, es necesario dedicar el tiempo y ser pacientes. El estudio presentado aplicó el condicionamiento operante durante 4 meses, pero los resultados comenzaron a observarse a la 2 semana del experimento, por lo que es factible indicar que, con más tiempo disponible para realizar esta práctica, podrían obtenerse mejores resultados.

El estrés, según (Chapman, 2003), aumenta en un gran porcentaje la susceptibilidad de los marsupiales a las enfermedades y, la necesidad de restringir y manipular a estos animales, puede provocarle al ejemplar lesiones graves e incluso la muerte(Poole, 1971), sin mencionar el peligro que involucra para los cuidadores y médicos veterinarios presentes. Martínez citado en (Damian, 2016) indica que el uso de condicionamiento operante con refuerzo positivo es una técnica con la cual se evita el uso de métodos restrictivos físicos y químicos que alteran el estado de bienestar de los animales, pudiendo ser útil en la detección de enfermedades de forma precoz, para su tratamiento. Por esta razón, la utilización del condicionamiento operante, puede ser una gran herramienta para reducir riesgos en el manejo de cualquier animal en cautiverio.

Respecto al nivel de confianza de los individuos, tres de estos que consumieron el tratamiento, uno es el macho alfa, otro es un macho juvenil y la última es una hembra pequeña de $10 \mathrm{~kg}$. Es importante mencionar que antes del régimen de condicionamiento operante el macho alfa ya se encontraba en un entrenamiento básico con target y de contacto libre. Además, este individuo, según es reportado por los cuidadores, siempre ha demostrado una sensibilidad baja a estímulos humanos, posiblemente por su naturaleza de alfa en el grupo de ejemplares.
En cuanto al macho juvenil y la hembra, los cuidadores reportan que siempre han sido curiosos ante la interacción humana, por lo que, al momento de llegar a la fase adulta, se encontraban bastante desensibilizados. Sin embargo, esto fue un comportamiento natural muy específico en estos animales, ya que, los otros ejemplares del grupo mantenían distancia con los cuidadores y presentaban tendencias de escape ante los movimientos repentinos. Además, como efecto colateral del condicionamiento aplicado a los ejemplares, los cuidadores reportaron una disminución muy marcada del tiempo de ingreso de los Ualabies a su dormitorio. Esta actividad, que anteriormente se realizaba en 1 hora y con la constante persecución de los individuos, fue disminuida a 15 minutos sin la necesidad de perseguir a ninguno.

Una de las limitaciones más grandes del estudio fue la falta de información previa para realizar el plan de condicionamiento, por lo que, este fue planteado en las bases de un condicionamiento más general. También se presentaron factores externos como cambio de cuidadores en ciertos días, ejemplares en celo, ruidos afuera del recinto como animales que se encuentran libres en el parque $\mathrm{o}$ el personal de limpieza; estas fueron situaciones que escapaban al control del estudio, pero su importancia en los resultados fue notoria durante el condicionamiento.

\section{Agradecimientos}

Al personal del Parque Zoológico Nacional La Aurora, por el apoyo y participación brindad as en elaboración del estudio.

\section{BibliografíA}

1. Chapman. (2003). The social behaviour and captive managemnet of Bennett's wallabies, Macropus rufrogriseus rufrogriseus. Australia: University of Tasmania.

2. Cheney, E. \&. (1980). Lithium-chloride.induced adversion in the opossum (Didelphis virginiana). Psysiological Psychology, 383-385. 
3. Crowell. (2008). Use of Operant Conditioning to Facilitate Examination of Zoo Animals. Compendium, 223.

4. Damian. (2016). Implementación de un programa de condicionamiento operante para manejos clínicos aplicado a felinos en cautiverio en el Parque Ecológico Ehécatl. Toluca, México: Universidad Autónoma del estado de México.

5. Emer, M. H. (2015). Predators in training: Operant conditioning of novel behavior in wild Burmese pythons (Python molurus bivittatus). Animal Cognition, 269-278.

6. Gomez, G. \&. (2015). Evaluación de un programa de condicionamiento operante con refuerzo positivo en un grupo de felinos (Panthera tigris) del parque zoológico Matecaña-Pereira-Colombia. Colombia: Universidad Tecnológica de Pereira.

7. Guzmán. (2018). Aspectos sanitarios, manejo clínico y entrenamiento del jaguar Panthera onca, (Linnaeus 1758) en Zoológicos. Bogota, D.C: Universidad de Ciencias Aplicadas y Ambientales.

8. Hellmuth, A. W. (2012). Using Operant Conditioning and Desensitization to Facilitate Veterinary Care with Captive Reptiles. ScienceDirect, 425-443.

9. Hincapié. (2019). Plan de Condicionamiento operante en Chimpancés (Pan troglodytes), para colecta de muestra sanguínea. Colombia: Universidad Tecnológica de Pereira.

10. Holden, G. W. (2006). Operant-conditioning programme for White rhinoceros, Black rhinoceros and Indian or Greater one-horned Asian rhinoceros Ceratotherium simum, Diceros bicornis and Rhinoceros unicornis at Whipsnade Wild Animal Park, Dunstable, UK. International Zoo Yearbook.

11. Hoyos. (2017). Diseño e implementación de un protocólo de entrenamiento con fines médicos en rinocerontes blancos (Ceratotherium simum, burchell 1817) mediante condicionamiento operante con refuerzo positivo en el Bioparque ukumarí, Pereira. Antioquia, Colombia: Corporación Universitaria Lasallista.

12. Jácome. (2012). Diseño de un plan de técnicas de condicionamiento animal (ECO), para la obtención de una respuesta clínica efectiva (RECE) en espécimen de jaguar (Panthera onca). Quito, Ecuador: Universidad central del Ecuador.

13. James. (1937). An experimental study of the defense mechanism in the opossum, with emphasis on natural behavior and its relation to mode of life. The pedagogical Seminary and Journal of Genetic Psychology, 95-100.

14. Martina, C. \& (2020). Exploring individual variation in associative learning abilities through an operant conditioning task in wild baboons. Plos one, 1-20.

15. Mattinson. (2012). Training Birds and Small Mammals for Medical Behaviors. Research Gate, 487-499.
16. Molina. (2018). Diseño y evaluación de un programa de condicionamiento operante para toma de muestra sanguínea, materia fecal y citología vaginal en dos hembra de rinoceronte blanco (Ceratotherium simum) del Bioparque Ukumari, Pereira-Colombia. Bogota, Colombia: Universidad de ciencias aplicadas y ambientales UDCA.

17. Ordóñez. (2012). Determinación de Helmintos Gastrointestinales en primates no humanos del parque zoológico Nacional La Aurora Guatemala, Guatemala. Guatemala, Guatemala: Universidad de San Carlos de Guatemala.

18. Panini. (1986). Psicologia comparadad de los marsupiales. Revista Latinoamericana de psicologia, 215-246.

19. Poole, C. \&. (1971). Keeping Kangaroos in captivity. International ZooYearbook, 5-12.

20. Ravizza, H. \&. (1969). Hearing in primitive mammals, 1: Opossum (Didelphis virginianus). The Journal of Auditory Research, 1-7.

21. Savastano, H. \&. (2003). The development of an Operant Conditioning Training Program for New World Primates at the Bronx Zoo. Journal of Applied Animal Welfare Science.

22. Uribe. (2019). Implementación y seguimiento de un plan de condicionamiento operante con refuerzo positivo para finalidades médicas en jirafas (Giraffa camelopardalis) en el bioparque Ukumari-pereiraColombia. Bucaramanga, Colombia: Universidad Cooperativa de Colombia.

23. Weiss. (2003). The use of Classical and Operant Conditioning in Training Aldabra Tortoises (Geochelone gigantea) for Venipuncture and other Husbandry issues. Researchgate, 33-38.

24. Yin. (2006). Understanding Behavior: "How Animals Learn Operant Conditioning". Compendium. 